
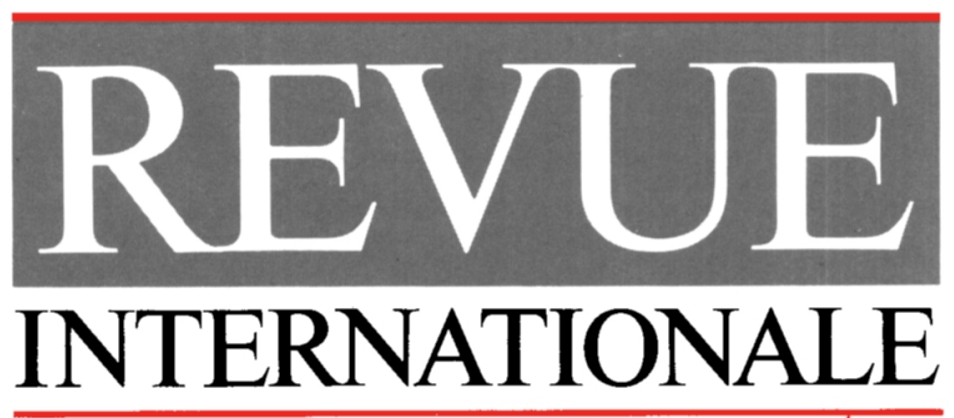

\title{
DE LA CROIX-ROUGE
}

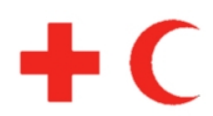

Publication bimestrielle du Comité international de la Croix-Rouge au service du Mouvement international de la Croix-Rouge et du Croissant-Rouge 


\section{COMITÉ INTERNATIONAL DE LA CROIX-ROUGE}

M. CORNELIO SOMMARUGA, docteur en droit de l'Université de Zurich, dr h.c. rer. pol. de l'Université de Fribourg (Suisse), dr h.c. en relations internationales de l'Université do Minho, Braga (Portugal), d' h.c. méd. de l'Université de Bologne (Italie), $d^{r}$ h.c. de l'Université de Nice-Sophia Antipolis, d $\mathrm{d}^{\mathrm{r}}$ h.c. en droit de l'Université nationale de Séoul (République de Corée), président (membre depuis 1986)

MM. PIERRE KELLER, docteur en philosophie en relations internationales (Yale), banquier, viceprésident (1984)

ERIC ROETHLISBERGER, docteur en sciences politiques de l'Institut universitaire de hautes études internationales de Genève, vice-président (1995)

ULRICH GAUDENZ MIDDENDORP, docteur en médecine, privat-docent de la faculté de médecine de l'Université de Zurich, ancien chef de la clinique chirurgicale de l'Hôpital cantonal, Winterthour (1973)

$M^{\text {me }}$ RENÉE GUISAN, secrétaire générale de l'«Institut de la Vie international», directrice d'établissements médico-sociaux vaudois, membre de l'«International Association for Volunteer Effort» (1986)

$\mathbf{M}^{\mathrm{me}} \quad$ ANNE PETITPIERRE, docteur en droit, avocate, professeure à la faculté de droit de l'Université de Genève (1987)

M. PAOLO BERNASCONI, avocat, lic. iur., chargé de cours de droit pénal économique aux Universités de Saint-Gall et de Zurich, ancien procureur général à Lugano, commissaire de la Fondation suisse Pro Juventute (1987)

$\mathrm{M}^{\mathrm{me}} \quad$ LISELOTTE KRAUS-GURNY, docteur en droit de l'Université de Zurich (1988)

$M^{\text {me }}$ SUSY BRUSCHWEILER, infirmière, ancienne directrice de l'Ecole supérieure d'enseignement infirmier de la Croix-Rouge suisse à Aarau, directrice générale SV-Service, restauration de collectivités (1988)

MM. JACQUES FORSTER, docteur en sciences économiques, professeur à l'Institut universitaire d'études du développement (IUED) à Genève (1988)

JACQUES MOREILLON, licencié en droit, docteur ès sciences politiques, secrétaire général de l'Organisation mondiale du Mouvement Scout, ancien directeur général au CICR (1988)

MAX DAETWYLER, licencié en sciences économiques et sociales de l'Université de Genève, «Scholar in Residence», International Management Institute (IMI) de Genève (1989)

RODOLPHE DE HALLER, docteur en médecine, privat-docent de la faculté de médecine de l'Université de Genève, président de l'Association suisse contre la tuberculose et les maladies pulmonaires (1991)

DANIEL THÜRER, docteur en droit, LL.M. (Cambridge) professeur ordinaire à l'Université de Zurich (1991)

$M^{\text {me }} \quad$ FRANCESCA POMETTA, licenciée ès lettres, ancien Ambassadeur de Suisse (1991)

MM. JEAN-FRANÇOIS AUBERT, docteur en droit, professeur à l'Université de Neuchâtel, ancien député au Conseil national et au Conseil des Etats (1993)

JOSEF FELDMANN, docteur ès lettres, professeur titulaire de l'Université de Saint-Gall, commandant de corps d'armée à disposition (1993)

$\mathrm{M}^{\mathrm{me}}$ LILIAN UCHTENHAGEN, docteur en sciences économiques de l'Université de Bâle, ancienne députée au Conseil national (1993)

M. GEORGES-ANDRÉ CUENDET, licencié en droit de l'Université de Genève, diplômé de l'Institut d'études politiques de l'Université de Paris, M.A. de l'Université de Stanford (USA), membre du Conseil administratif de Cologny/GE (1993)

M. ERNST A. BRUGGER, docteur en sciences naturelles, directeur de FUNDES (Fondation pour la promotion et le développement durable de petites entreprises), conseiller économique, professeur titulaire de l'Université de Zurich (1995)

\section{CONSEIL EXÉCUTIF}

\section{CORNELIO SOMMARUGA, président}

M. ERIC ROETHLISBERGER, vice-président permanent

M. JACQUES FORSTER, membre du CICR

$\mathrm{M}^{\mathrm{me}}$ ANNE PETITPIERRE, membre du CICR

M. PETER FUCHS, directeur général

M. JEAN DE COURTEN, directeur des Opérations

M. YVES SANDOZ, directeur de la Doctrine, du Droit et des Relations avec le Mouvement 


\title{
REVUE INTERNATIONALE DE LA CROIX-ROUGE
}

\section{SOMMAIRE}

JANVIER-FÉVRIER 1996

No 817

\author{
Spécial \\ XXVI ${ }^{\mathrm{e}}$ CONFÉRENCE INTERNATIONALE \\ DE LA CROIX-ROUGE ET DU CROISSANT-ROUGE \\ (Genève, 3-7 décembre 1995)
}

Editorial: Réflexion sur la XXVI ${ }^{e}$ Conférence internationale par Yves Sandoz, directeur, Droit, Doctrine et Relations avec le Mouvement

XXVI ${ }^{\mathrm{e}}$ Conférence internationale de la Croix-Rouge et du CroissantRouge (Genève, 3-7 décembre 1995).

- La cérémonie d'ouverture (3 décembre 1995)

- Première séance plénière (4 décembre 1995)

\section{LES DÉFIS HUMANITAIRES À L'AUBE DU XXI' SIÈCLE}

Déclaration de M. Mario Villarroel Lander, président de la Fédération internationale des Sociétés de la Croix-Rouge et du Croissant-Rouge .....

Déclaration de M. Cornelio Sommaruga, président du Comité international de la Croix-Rouge 
- Commission I - Victimes de la guerre et respect du droit international humanitaire

- Commission II - Valeurs humanitaires et réponses aux crises ....

- Deuxième séance plénière - Election de cinq membres de la Commission permanente (6 décembre 1995)

- Troisième séance plénière - Rapports des Commissions et adoption des résolutions (7 décembre 1995)

XXVI CONFÉRENCE INTERNATIONALE DE LA CROIXROUGE ET DU CROISSANT-ROUGE - RÉSOLUTIONS (ET ANNEXES)

Conseil des Délégués (Genève, $1^{\text {er }}$ et 2 décembre 1995) ...................... 138

Résolutions du Conseil des Délégués ................................................... 147

\section{COMITÉ INTERNATIONAL DE LA CROIX-ROUGE}

RÉDACTION DE LA REVUE - Jacques Meurant prend sa retraite 161

Nouveau rédacteur en chef de la Revue ........................................... 163

Vu l'abondance des matières, les rubriques «Faits et documents» (dont le tableau au 31 décembre 1995 de l'état des ratifications, adhésions et successions aux Conventions de Genève et aux Protocoles additionnels) et «Livres et revues» sont reportées au numéro de mars-avril 1996. La Revue poursuivra dans ce même numéro la publication de documents particulièrement significatifs de la XXVI ${ }^{e}$ Conférence internationale et un compte rendu de la $\mathrm{X}^{\mathrm{e}}$ session de l'Assemblée générale de la Fédération internationale des Sociétés de la Croix-Rouge et du Croissant-Rouge (Genève, 27-29 novembre 1995). 